\title{
Caroline Welsh
}

\section{Das Gehirn in Wissenschaft und Gegenwartsliteratur}

\section{Alternativen zum neurobiologischen Konstruktivismus}

\begin{abstract}
Der Beitrag untersucht wissenschaftliche und literarische Gegenentwürfe zum neurobiologischen Konstruktivismus und fragt nach poetologischen und anthropologischen Implikationen der Hirnforschung. Der erste Teil hebt anhand von Thomas Fuchs' Kritik am cartesianischen Erbe der kognitiven Neurowissenschaften und seiner Neuverortung von Subjektivität innerhalb des lebenden Organismus sowie den Ausführungen von Aris Fioretos und Durs Grünbein zur Literatur als ,alternativer Hirnforschung، die Bedeutung der subjektiven Innenperspektive für die Einbettung neurobiologischen Wissens über das Gehirn in anthropologisch relevante Kontexte hervor. Im zweiten Teil werden zwei Gehirngedichte Grünbeins aus der Schädelbasislektion wissensgeschichtlich aus ihrer bisherigen Zuordnung in den Kontext des neurobiologischen Konstruktivismus herausgelöst und zur Erforschung der Bedeutung subkortikaler Hirnareale im Rahmen der Neurochemie und Psychopharmakologie der 1980er Jahre in Beziehung gesetzt.
\end{abstract}

\section{Einleitung}

Mit Blick auf ihre Konsequenzen für unsere Selbstwahrnehmung und unser Menschenbild zieht die Hirnforschung wie kaum ein anderer Bereich naturwissenschaftlicher Grundlagenforschung das öffentliche Interesse auf sich. Renommierte Neurobiologen beanspruchen in populärwissenschaftlichen und neurophilosophischen Publikationen über die engeren Grenzen ihrer Wissenschaft hinaus die Deutungshoheit bei der Erforschung des Bewusstseins, des Denkens, Fühlens, Wollens und Handelns. Sie erklären sich zuständig nicht nur für die Anatomie, Neurologie und Pathologie des Gehirns, sondern auch für Fragen des Subjektiv-Seelischen. ${ }^{1}$

\footnotetext{
1 Stellvertretend Gerhard Roth: Das Gehirn und seine Wirklichkeit. Kognitive Neurobiologie und ihre philosophischen Konsequenzen. Frankfurt a. M.: Suhrkamp, 1994; Wolfgang Singer: Selbsterfahrung und neurobiologische Fremdbestimmung. Zwei konfliktträchtige Erkenntnisquellen. In: Deutsche Zeitschrift für Philosophie (2004). S. 235-255.
} 
Mit der Rezeption neurobiologischer Forschungsergebnisse in anderen Wissensbereichen ist in den letzten Jahrzehnten zudem eine ,heiße Zone des Wissens entstanden, die auf den ersten Blick quer steht, sowohl zu den etablierten Disziplinen als auch zur Trennung zwischen den Natur- und Geisteswissenschaften: In dieser Zone findet man bereits seit Längerem die Neuropsychologie und die Neurolinguistik, seit Kürzerem aber auch die Neurodidaktik, die Neurophilosophie, die Neuroethik, die Neurogeschichte und das Neurorecht. Auch die Literaturwissenschaft ist als kognitive Literaturwissenschaft und in der Neuroästhetik vertreten. ${ }^{2}$ Neben der Erschließung neuer und wichtiger Fragehorizonte geht es in dieser Form der Interdisziplinarität darum, bisher geistes- und kulturwissenschaftliche Fragestellungen auf der Basis des Wissens der Hirnforschung neu zu beantworten bzw. bereits vorhandene Theorien mit einer naturwissenschaftlichen Autorität auszustatten, indem man sie neurowissenschaftlich umformuliert. ${ }^{3}$

Der folgende Beitrag ${ }^{4}$ versteht unter einer interdisziplinären Zusammenführung von Literatur und Hirnforschung nicht die Übernahme naturwissenschaftlicher Methoden und Theorien in den Bereich der Literatur- und Kulturwissenschaft. Solche Forschungen leisten zwar dem Aufruf der Hirnforscher an die Geisteswissenschaftler im „Manifest“ zur Hirnforschung von 2004 Folge, „gemeinsam ein neues Menschenbild zu entwerfen“",5 sie bestätigen jedoch zugleich den aktuellen Anspruch der Hirnforschung in ihrer Ausbreitung auch auf das Feld der Geisteswissenschaften.

2 Vgl. Karin Herrmann (Hrsg.): Neuroästhetik. Perspektiven auf ein interdisziplinäres Forschungsgebiet. Kassel: University Press, 2011; Karl Eibl, Katja Mellmann und Rüdiger Zymner (Hrsg.): Im Rücken der Kulturen. Paderborn: Mentis, 2007; Raoul Schrott und Arthur Jacobs: Gehirn und Gedicht. Wie wir unsere Wirklichkeiten konstruieren. München: Hanser, 2011; Gerhard Lauer: Das Spiel der Einbildungskraft. Zur kognitiven Modellierung von Nachahmung, Spiel und Fiktion. In: Literatur als Spiel. Evolutionsbiologische, ästhetische und pädagogische Konzepte. Hrsg. von Thomas Anz und Heinrich Kaulen. Berlin: De Gruyter, 2009. S. 27-38; Kilian Koepsell und Carlos Spoerhase: Neuroscience and the Study of Literature. Some Thoughts on the Possibility of Transferring Knowledge. In: Journal of Literary Theory 2 (2008). S. 363-374.

3 Das aktuelle Interesse an neurokognitivistischen Ansätzen in der Linguistik und Ästhetik spiegelt sich in der Gründung neuer Institute der Max-Planck-Gesellschaft: dem MPI für Kognitionsund Neurowissenschaften in Leipzig unter der Leitung der Neurolinguistin Prof. Angela Friederici (gegründet 2004) und dem MPI für empirische Ästhetik, Frankfurt a. M., unter der Leitung des Literaturwissenschaftlers Winfried Menninghaus (gegründet 2013).

4 Der Beitrag wurde im Rahmen meiner Tätigkeit als Senior Fellow im Erlanger EFI-Projekt „Human Rights in Healthcare" fertiggestellt.

5 Hannah Monyer u. a.: Das Manifest. Elf führende Neurowissenschaftler über Gegenwart und Zukunft der Hirnforschung. In: Gehirn \& Geist 6 (2004). S. 30-37. 
Wichtiger und auch schwieriger $\mathrm{zu}$ beantworten, erscheint mir die Frage nach dem Beitrag der Literatur zur kulturellen Einbettung und kritischen Reflexion naturwissenschaftlicher Erkenntnisse, die im Falle der Hirnforschung das Bild vom Menschen direkt betreffen. Wie positioniert sich die Literatur im Verhältnis zum Expertenwissen der Neurobiologie? Reagieren literarische Texte in erster Linie auf den umfassenden Deutungsanspruch der Hirnforschung in neurophilosophischen Abhandlungen, auf Texte also, die sich selbst bereits im Grenzbereich zwischen Naturwissenschaft und Philosophie bewegen? Oder setzen sie sich detaillierter mit spezifischen neurobiologischen Fragestellungen und Forschungsergebnissen auseinander? Wenn ja, aus welchen Quellen beziehen sie ihr Wissen? Führt die Ästhetisierung naturwissenschaftlichen Wissens („Singende Hirne [...] sind wir"6), seine Einbettung in andere kulturelle Kontexte, auch zu einer Modifikation des mit der Hirnforschung verbundenen Menschenbildes, zu einem neuen Blick auf das eigene, „babylonische Gehirn“?7 Inwiefern sind gerade literarische Texte dazu geeignet, gesellschaftliche und anthropologische Konsequenzen der Hirnforschung kritisch zu reflektieren? Welche Bedeutung hat schließlich das Wissen um die Neurobiologie des Gehirns für zeitgenössische Dichtungstheorien?

Welche Möglichkeiten der Hirnforschung einmal praktisch umgesetzt werden, welche Vorstellung wir in Zukunft vom Menschen haben werden, hängt nicht nur von den empirisch-neurobiologischen Ergebnissen der Hirnforschung ab, sondern ebenso sehr davon, welchen Stellenwert dieses Wissen sowohl innerhalb einer naturwissenschaftlichen Anthropologie als auch im Zusammenspiel mit anderen Wissensformen einnehmen wird und wie ihre Ergebnisse vor diesem Hintergrund in die breitere Kultur eingebettet werden. Um solche Einbettungen neurobiologischen Wissens über das Gehirn in größere, anthropologisch relevante Kontexte wird es im ersten Hauptteil des Aufsatzes gehen: innerwissenschaftlich zunächst mit der Darstellung des phänomenologisch-ökologischen Ansatzes von Thomas Fuchs und seiner Kritik am neurobiologischen Reduktionismus der kognitiven Neurowissenschaften. Sodann mit Blick auf die Selbstpositionierung der Literatur als Erkundung des Gehirns aus der subjektiven Innenperspektive in einigen poetologischen Essays von Aris Fioretos und Durs Grünbein. Abschließend werden Möglichkeiten und Effekte einer Zusammenführung inkommensurabler Menschenbilder in einem Gedicht aus Grünbeins Schädelbasislektion (1991) analysiert.

6 Durs Grünbein: Zerebralis. In: ders.: Schädelbasislektion. Gedichte. Frankfurt a. M.: Suhrkamp, 1991. S. 134-136, hier S. 136. Herv. im Orig.

7 Durs Grünbein: Mein babylonisches Gehirn. In: ders.: Gedicht und Geheimnis. Aufsätze 19902006. Frankfurt a. M.: Suhrkamp, 2007. S. 19-33. 
Der zweite Hauptteil hinterfragt die in der Literaturwissenschaft postulierte Bedeutung des neurobiologischen Konstruktivismus in den Gehirngedichten Grünbeins und unternimmt eine historisch präzisere wissensgeschichtliche Verortung zweier Gedichte aus der Schädelbasislektion. Der wissensgeschichtliche Kontext dieser Gedichte ist, so die These, nicht wie bisher postuliert die kognitive Neurowissenschaft und Neurophilosophie, sondern die in den 1980er Jahren aktuelle Forschung zur Wirkungsweise von Psychopharmaka und Neurotransmittern bei der neuronalen Informationsweiterleitung. Es sind die in dieser Forschung fokussierten subkortikalen Areale des Gehirns in der Nähe der Schädelbasis (nicht die Großhirnrinde), die Grünbein auch in seiner Theorie zur poetischen Kreativität interessieren - was nicht gleichbedeutend ist mit der Überzeugung, dass die Hirnforschung im Alleingang darauf eine befriedigende Antwort finden könnte.

\section{Wissenschaftliche und literarische Einbettungen neurobiologischen Wissens}

\section{Der neurobiologische Reduktionismus und seine wissenschaftlichen Alternativen}

In neurophilosophischen und populärwissenschaftlichen Abhandlungen haben prominente Hirnforscher seit den 1990er Jahren gezielt Einfluss auf die breitere kulturelle Einbettung ihrer neurobiologischen Forschungsergebnisse genommen. Einige Titel machen deutlich genug, dass sie es auf eine Veränderung des Menschenbilds anlegen, wobei allerdings der Bereich neurophilosophischer Hypothesenbildung deutlich in Richtung Spekulation überschritten wird - Francis Crick: The Astonishing Hypothesis. The Scientific Search for the Soul; Gerhard Roth: Wie das Gehirn die Seele macht; Wolf Singer: Keiner kann anders, als er ist. Verschaltungen legen uns fest. Wir sollten aufhören von Freiheit zu reden. ${ }^{8}$ Auf der Ebene der Popularisierung wissenschaftlichen Wissens durch prominente Neurowissenschaftler wird die Materialität des Geistes und der Seele mit einem Pathos der Desillusionierung ausgerufen und der Mensch auf sein Gehirn reduziert. Aus neu-

8 Wolf Singer: Keiner kann anders, als er ist. http://www.faz.net/aktuell/feuilleton/hirnforschungkeiner-kann-anders-als-er-ist-1147780.html. Frankfurter Allgemeine Zeitung Online, 8. Januar 2004. (14. April 2015); Francis Crick: The Astonishing Hypothesis. The Scientific Search for the Soul. New York: Simon \& Schuster, 1994; Gerhard Roth: Wie das Gehirn die Seele macht. Frankfurt a. M.: Suhrkamp, 2001. 
robiologischer Perspektive gibt es, so der Stand der neurophilosophischen Hypothesen, keine Willensfreiheit; das Ich wird als Basis des Bewusstseins, die subjektive Selbstwahrnehmung in der 1. Person Singular, zu einem Epiphänomen neuronaler Prozesse, zu einem Gehirnkonstrukt, das selbst keinen Einfluss, keine ,Wirkungsmacht' auf neurobiologische Vorgänge ausübt. ${ }^{9}$

Die Faszination dieser Arbeiten und ihre Resonanz in der Öffentlichkeit scheint etwas mit dem zu tun zu haben, was der Schriftsteller Gottfried Benn bereits vor 100 Jahren mit Bezug auf die damaligen Ergebnisse der Hirnforschung konstatiert und in seinen Rönne-Novellen hinsichtlich der Auswirkungen auf das Menschenbild ausgekundschaftet hat:

\begin{abstract}
Was die Resultate in ihrer Gesamtheit bedeuteten, war mehr als eine völlig neue Erkenntnis von der Bedeutung der nervösen Organe; vielmehr handelte es sich um dies: man hatte an den Geweben des menschlichen Körpers experimentiert und hatte Reaktionen bekommen aus dem Gebiet des Seelischen; man hatte sich während der Arbeit mitten im Bereich der Physiologie dem Psychischen gegenüber gesehen; man war zu der Stelle gekommen, da waren die beiden Lebensbereiche zusammengeknotet und man konnte von hier aus in das dunkle rätselhafte Reich des Psychischen tasten. Und damit stand man vor etwas unerhört Neuem in der Geschichte der Wissenschaften. ${ }^{10}$
\end{abstract}

Wissensgeschichtlich ist der Gedanke von der Materialisierung der Seele so neu also nicht, wie heutige Hirnforscher behaupten. Was zu Benns Zeiten als etwas „unerhört“ Neues empfunden wurde, dass „das Gehirn psychische Phänomene aus rein materiellen Grundlagen erzeugt ", ${ }^{11}$ wird heute in akademischen Kreisen kaum jemand ernsthaft bestreiten. Die Kontroversen fangen dort an, wo es um konkrete wissenschaftliche Fragestellungen, Methoden der Hirnforschung und allgemeine anthropologische Schlussfolgerungen geht. Besonders virulent sind diese dort, wo es um Fragen der Konzeptionen von Bewusstsein, Subjekt, Subjektivität und lebensweltlicher Selbst- und Welterfahrung geht.

In den letzten Jahren hat sich eine ,kritische Neurowissenschaft‘ zum Ziel gesetzt, reduktionistischen Erklärungsansätzen durch die Einbettung neurobiologischer Forschungsergebnisse in einen breiteren interdisziplinären Kontext

9 Roth: Das Gehirn und seine Wirklichkeit, S. 253.

10 Gottfried Benn: Beitrag zur Geschichte der Psychiatrie [1910]. In: ders.: Sämtliche Werke. Bd. 3. Hrsg. von Gerhard Schuster. Stuttgart: Klett-Cotta, 1987. S. 8-9. Zu Benns Darstellung der Konsequenzen eines auf die Funktionsweise des Gehirns reduzierten Menschenbilds vgl. Caroline Welsh: Die „Dunkelheit hinter dem Stirnportal“. Begegnungen von Literatur und Hirnforschung. In: Hirngespinste. Denkprozesse zwischen Störung, Genialität und Fiktionalität in Künsten und Wissenschaften. Hrsg. von Wladimir Velminski. München: Fink, 2013. S. 63-80.

11 Thomas Fuchs: Das Gehirn - ein Beziehungsorgan. Eine phänomenologisch-ökologische Konzeption. Stuttgart: Kohlhammer, 2013. S. 19. 
entgegenzuwirken. ${ }^{12}$ Eine grundlegende Kritik am idealistischen Erbe der kognitiven Neurowissenschaft leistet Thomas Fuchs in seiner Monographie Das Gehirn - ein Beziehungsorgan. ${ }^{13}$ Mit seiner Konzeption des Gehirns als ein in den Gesamtorganismus eingebettetes „Beziehungsorgan“ bietet er zugleich einen umfassenden Gegenentwurf, der neurobiologische Theorien zur Funktionsweise insbesondere subkortikaler Hirnareale und phänomenologischer Ansätze miteinander kombiniert. Die in den kognitiven Neurowissenschaften beliebten neurokonstruktivistischen Theorien zur Arbeitsweise des (Groß-)Hirns sind, so Fuchs, keine Alternative zum cartesianischen Dualismus, sondern im Gegenteil lediglich eine Materialisierung des cartesianischen Subjekts unter Beibehaltung grundlegender, nicht hinterfragter Denkmuster.

In beiden Fällen werde erstens ein autonomes übergeordnetes System - die immaterielle Seele der cartesianischen res cogitans oder aber das Gehirn - dem Körper bzw. dem ,Restkörper abzüglich des Gehirns und der Umwelt radikal gegenübergestellt. Der Körper selbst, seine eigene Befindlichkeit ebenso wie die über die Sinnesorgane vermittelten Informationen über die Umwelt erschienen lediglich als ,Repräsentationen` innerhalb der übergeordneten Systeme ,Seele`/ ,Gehirn'.

Auf der Basis dieser Repräsentationen würden die Daten aus dem Körper (und der Umwelt) erfasst und verarbeitet, wobei das Gehirn zweitens alle Aufgaben übernehme, die zuvor dem cartesianischen Ich zugesprochen wurden: Es denkt, berechnet, entscheidet, fühlt, handelt, wenngleich nicht mehr unter den Bedingungen der Freiheit, sondern der den Naturgesetzen unterstehenden Neuronenverschaltungen. Diese einfache Substitution des cartesianischen Subjekts durch das Gehirn spiegele sich auch im Sprachgebrauch: Auch auf der Ebene der Syntax tritt das Gehirn an die Stelle des grammatikalischen Subjekts. ${ }^{14}$

An einem weiteren Kritikpunkt deutet sich zugleich an, wo Fuchs das Denkmuster zu durchbrechen und mit seinem Gegenentwurf anzusetzen gedenkt: Bewusstsein, Subjektivität und subjektives Erleben in der 1. Person Singular würden in beiden Modellen, wenngleich jeweils auf unterschiedliche Weise, radikal vom

12 Jan Slaby: Perspektiven einer kritischen Philosophie der Neurowissenschaften. In: Deutsche Zeitschrift für Philosophie 59 (2011). S. 375-390; Suparna Choudhury und Jan Slaby (Hrsg.): Critical Neuroscience. A Handbook of the Social and Cultural Contexts of Neuroscience. Chichester: Blackwell, 2012; Felix Hasler: Neuromythologie. Eine Streitschrift gegen die Deutungsmacht der Hirnforschung. Bielefeld: transcript, 2012; Markus Stier: Ethische Probleme in der Neuromedizin. Identität und Autonomie in Forschung und Diagnostik. Frankfurt a. M.: Campus, 2006.

13 Fuchs: Gehirn - Beziehungsorgan, S. 25-92. Fuchs ist Psychiater und Ko-Direktor des Interdisziplinären Forums für Biomedizin und Kulturwissenschaften in Heidelberg.

14 Ebd., S. 65-68. Zum Folgenden vgl. ebd., S. 77-92. 
lebendigen Organismus getrennt. Der Cartesianismus ordne sie als für das Denken und Handeln entscheidende Qualitäten der immateriellen Seele zu, die kognitive Neurowissenschaft betrachte sie als Epiphänome neuronaler Gehirnprozesse ohne eigene Wirkmacht. Ein theoretisches Gegenmodell, das für „eine adäquate Interpretation neurobiologischer Daten " hinsichtlich ihrer Bedeutung für das Verständnis von Bewusstsein und Subjektivität tauglich sei, müsse demgegenüber, so Fuchs, mit der „lebensweltlichen Erfahrung kompatibel“ sein. Es müsse „von der 1. und 2. Person, also der Selbsterfahrung von lebendigen Personen ausgehen“ und den Körper als lebenden Organismus miteinbeziehen. ${ }^{15}$ Hier stieße die Naturwissenschaft allerdings an ihre Grenzen, weil die Voraussetzung ihres Erfolgs in der Objektwelt gerade im Ausschluss des Subjektiven bestehe.

Fuchs' Gegenmodell besteht in einer Neuverortung der Subjektivität innerhalb des lebenden Organismus, der nun verstanden wird als zugleich biologischer Körper und „subjektiver Leib“. Im Anschluss an die Phänomenologie betont er die Bedeutung der „leiblichen Subjektivität“, mit dem der „im Hintergrund ,gelebte، Leib, als Ort diffusen Befindens [...] [,] als Resonanzraum aller Stimmungen und Gefühle [...] und Medium aller Wahrnehmungen, Bewegungen und Handlungen" 16 gemeint ist. Personale Subjektivität und Bewusstsein sind somit notwendig „verkörperte Subjektivität“, Erfahrung des Selbst und der Welt durch das Medium des Leibes in seiner jeweils spezifischen Gesamtbefindlichkeit. Diese phänomenologische Grundannahme von der leiblichen Grundlage der Subjektivität und des Bewusstseins findet

ihre Entsprechung in den Verhältnissen auf der neurobiologischer Ebene: Bewusstsein entsteht auf der Basis der Interaktion von Körper und Gehirn, und zwar so, dass der Körper nicht nur sekundär zu seinem Gegenstand wird, sondern für seinen Aufbau selbst konstitutiv ist. ${ }^{17}$

Um diese Zentralthese zu belegen, verknüpft Fuchs neurobiologische Thesen zur Funktion subkortikaler Hirnareale bei der Regulierung autonomer Körperpro$z^{z e s s e}{ }^{18}$ mit darauf aufbauenden neurobiologischen Bewusstseinskonzeptionen

15 Ebd., S. 92.

16 Ebd., S. 97. Herv. im Orig.

17 Ebd., S. 137.

18 Ebd., S. 135: „Die primäre Funktion des Gehirns ist die Regulation des inneren Milieus [...]. Hirnstamm und Zwischenhirn, insbesondere der Hypothalamus, sind zentrale Steuerungsorgane für neuroendokrine, viszerale und immunologische Prozesse. Sie regulieren Atmung, Kreislauf [...] und eine Reihe weiterer autonomer Körperprozesse. Zentrale und periphere Funktionen sind dabei in komplexen, horizontal-vertikalen Regelkreisen verknüpft, die z .B. die Hormon-, Glukose-, Sauerstoff- oder Kohlendioxid-Konzentration im Blut regulieren. Bereits auf dieser Ebene lässt sich keine Trennlinie zwischen Gehirn und extrazerebralem Körper ziehen.“ 
von António Damásio und Jaak Panksepp. ${ }^{19}$ An der „Wurzel des Bewusstseins“ liegen demnach Regulationsprozesse, die das innere Milieu des Körpers betreffen: Verschiedene Subsysteme des Gehirns verarbeiten „neuronale und humorale Signale aus dem Körper und integrieren sie zu einer ,Körperlandschaft', die sich ständig modifiziert.“ Letztere bildet den „physischen Zustand des Organismus“ ab und stellt nach Damásio das „Protoselbst“, eine Vorstufe des Bewusstseins, dar. Das „Kernbewusstsein“, verstanden als bewusst wahrgenommenes elementares Lebensgefühl, entsteht darauf aufbauend im Zusammenspiel zwischen subkortikalen (u. a. des Thalamus), und kortikalen Hirnstrukturen. Konzeptionell tritt hier an die Stelle der Repräsentation des Körpers im Gehirn die Resonanz zwischen dem Gehirn und den „gesamtorganismischen Zuständen“. ${ }^{20}$ Die Verschiebung vom Repräsentationsmodell zum Resonanzmodell erlaubt eine theoretische Konzeption der Interaktion zwischen Körper und Gehirn auf der Basis gleichwertiger, miteinander in Wechselwirkung stehender Systeme. So wird einsichtig, wie ein subjektiv erlebter Leib selbst konstitutiv am Aufbau von Bewusstsein beteiligt sein kann. Die cartesianische Trennung zwischen einem autonomen übergeordneten System und dem (Rest-)Körper wird aufgehoben, das Gehirn „als Organ eines Organismus in seiner Umwelt“ integriert. Als „Beziehungsorgan“ vermittelt es „unsere Beziehungen zur Welt, zu anderen Menschen und zu uns selbst“"21 - aber es bleibt Organ der Vermittlung auf der Grundlage fortlaufender Rückkopplungen zwischen Gehirn, Organismus und Umwelt. ${ }^{22}$

\section{Literarische Kranionautik als alternative Hirnforschung bei Fioretos und Grünbein}

Was wir Prosa nennen, ist in Wirklichkeit rhythmisierte Hirntätigkeit, das Rein-und-RausSpiel des Bewusstseins, das im Versteck der Sprache umherjagt wie in seinem Bau Kafkas Tier. Momentweise nur fällt ins Dunkel des Schädels ein Streifen Licht. ${ }^{23}$

Die Nähe der Literatur zur Hirnforschung ergibt sich aus dem gemeinsamen Interesse an der „Dunkelheit hinter dem Stirnportal“ . ${ }^{44}$ Sie unterscheiden sich zugleich erheblich in ihren Erkundungsformen und in den Antworten, die sie pro-

19 Ebd., S. 137. Ebenso die folgenden vier Zitate.

20 Ebd., S. 138.

21 Ebd., S. 21.

22 Ebd., S. 144-152.

23 Durs Grünbein: Unter uns Hunden. In: Aris Fioretos: Mein schwarzer Schädel. Berlin: DAAD Berliner Künstlerprogramm, 2003 [Spurensicherung, Bd. 14]. S. 57-61, hier S. 60.

24 Aris Fioretos: Mein schwarzer Schädel, S. 21. 
duzieren. Während die Literatur sich seit dem späten achtzehnten Jahrhundert zur Spezialistin für die Erkundung und Darstellung subjektiver innerer Erlebniszustände mit den Mitteln der Sprache entwickelt hat, gilt der Blick des Hirnforschers dem Gehirn als Objekt naturwissenschaftlicher Erforschung. Der literarische und der wissenschaftliche Blick auf das Gehirn stehen somit stellvertretend für zwei bisher grundsätzlich inkommensurable Perspektiven. Ihre Erkenntnisse sind mit den Worten Durs Grünbeins aus seinem Nachwort zu Aris Fioretos' Essay Mein schwarzer Schädel:

[e]ntweder Ausdruck, Fetzen von Selbsterkenntnis oder Neurochemie, Geheimkode der Stoffwechselprozesse im Hirn. Dazwischen gibt es nichts als die Grauzone, ein Niemandsland, das sich der Sprache entzieht. ${ }^{25}$

Fioretos und Grünbein legen es darauf an, dieses „Dazwischen“ zwischen Neurowissenschaft und erlebter Innenperspektive zu erkunden. Der ,schwarze Schädel $^{`}$, die ,Schädelbasis` benennen dabei den Ort der Erkundungen, ohne sich auf eine der beiden Perspektiven festzulegen:

\footnotetext{
Was uns verbindet, ist eine fixe Idee. Fast zwanghaft kreist unser Gespräch, das wir seit acht Jahren führen, um jenen blinden Fleck, der als Seele vorzeiten die Dichter beschäftigte und den man heute, ebenso vage, mit denselben schwammigen Assoziationen, das Gehirn nennt. Fioretos spricht von der Dunkelheit im eigenen Kopf. Dichtung, soviel steht für uns beide fest, ist der Versuch mittelst Schriftsprache Licht in das Dunkel zu bringen. ${ }^{26}$
}

Der Alleinanspruch der Hirnforschung auf die Erkundung des Gehirns wird hier deutlich relativiert, die Dichtung als alternative Hirnforschung, als adäquater Ausdruck des Bewusstseins reflektiert: Begriffe wie ,Seele‘ und ,Gehirn“ erscheinen als gleichwertige Umschreibungen eines blinden Flecks, wobei an die Stelle eines wissenschaftlichen Fortschritts das gemeinsame Assoziationsfeld der Begriffe und das bleibende Enigma des Schädelinnenraums treten. Nicht die modernen bildgebenden Verfahren der Hirnforschung, sondern die Schriftsprache wird als geeignetes Medium zur Beleuchtung des Schädelinnenraums präsentiert. In „Hirnbilder und Denkschriften“, einem Dialog zwischen Grünbein und Michael Hagner, reflektiert Grünbein eine gute Dekade nach diesem Aufsatz noch einmal das Verhältnis von Dichtung und Hirnforschung. Der neurowissen-

25 Grünbein: Unter uns Hunden, S. 60.

26 Ebd., S. 60. Zu Grünbeins und Fioretos' öffentlich inszeniertem Dialog vgl. Joachim Grage: Stockholm gris - Gehirn, Stadt und Text bei Aris Fioretos und Durs Grünbein. In: Skandinavistik 34.2 (2004). S. 133-149. 
schaftlichen Tranceforschung entnimmt er den Begriff der „Verarbeitungstiefe“27, um mit ihm gerade die „Autonomie der Phantasie“ 28 im Zustand schöpferischer Imagination zu begründen. Ebenso wie sich im Zustand der Trance das Gehirn „auf einem prinzipiell anderen Niveau geistiger Regsamkeit“29 bewege, zeichne sich auch der Zustand literarischer sowie wissenschaftlicher Kreativität ${ }^{30}$ durch eine „erhöhte“"31 und „umfassende Hirnaktivität“32 aus, die es der Imagination ermögliche, sich dank „komplexer assoziativer Verarbeitungsprozesse [...] aus den Fesseln der Konventionen [zu] befreien“33 und „etwas Neues zu schaffen““. ${ }^{34}$ Nicht die „Hirnbilder“ bildgebender Verfahren sondern die „Denkschriften“ der Dichtung erweisen sich in diesem Dialog als Ort, an dem sich „die Verarbeitungsprozesse im Gehirn während des sogenannten schöpferischen Arbeitens“35 studieren lassen. ${ }^{36}$

Fioretos hat für diese Art literarischer Erkundungen den Neologismus des „Prosaisten als Kranionauten“ geprägt. Als Astronaut im Universum des Gehirns, hat es sich dieser zur Aufgabe gemacht, mittels Introspektion „dem eigenen Gehirn in die Karten zu gucken. ${ }^{37}$ Analog heißt es dazu in Grünbeins lyriktheoretischem Essay „Mein babylonisches Gehirn“ über den Lyriker in dem Moment, wo die alltäglichen semantischen Bindungen sich lockern und sich die Möglichkeit neuer semantischer Kombinationen und poetischer Verdichtungen eröffnet: „Es ist, als würde er seinem Hirn bei der Arbeit zusehen. “" ${ }^{38}$ Beide veranschaulichen

27 Durs Grünbein und Michael Hagner: Hirnbilder und Denkschriften. Ein einführender Dialog. In: Hirngespinste. Denkprozesse zwischen Störung, Genialität und Fiktionalität in Künsten und Wissenschaften. Hrsg. von Wladimir Velminski. München: Fink, 2013. S. 21-45, hier S. 22 und 29-30.

28 Ebd., S. 41.

29 Ebd., S. 23.

30 Ebd., S. 34-35.

31 Ebd., S. 39.

32 Ebd., S. 23. Ebenso auch das folgende Zitat.

33 Ebd., S. 41.

34 Ebd., S. 40.

35 Ebd., S. 33-34.

36 Ebd., S. 22: „Es ist eine Illusion, zu glauben, man könne dem Subjekt, diesem Herrn Cogito des René Descartes, bei der Arbeit zusehen, indem man eine Versuchsperson in die Röhre schiebt und nachher auf dem Computer die Bilder der Magnetresonanztomographie auswertet. Von den graphischen Abstraktionen auf wirkliche Wahrnehmungs- und Denkprozesse, erst recht schöpferische Aktivitäten des Hirns, schließen zu wollen, ist einfach albern." Herv. im Orig.

37 Fioretos: Mein schwarzer Schädel, S. 9.

38 Grünbein: Mein babylonisches Hirn, S. 21. 
das Verfahren der Introspektion topographisch als Begegnung des Schreibenden mit der Fremdheit des eigenen Gehirns. ${ }^{39}$

Die Methode der Introspektion hat sich spätestens seit Karl Philipp Moritz' Aufforderung zu distanzierter Selbstbeobachtung sowohl in der Literatur als auch in der Psychologie etabliert. Seit der literarischen Moderne (und in diese Tradition ordnet sich Fioretos auch ein) entwickelt die Literatur Darstellungsformen, um das Bewusstsein ,in flagranti ‘ zu ertappen, Darstellungsformen, die sich an den zeitgenössischen Wissenschaften orientieren. Auch fingierte Reisen durch innere Gehirnlandschaften haben literarische Tradition. ${ }^{40}$ Wichtig ist nicht die Neuheit dieser Methode, sondern die Positionsbestimmung der Literatur hinsichtlich ihres spezifischen Beitrags zur aktuellen Anthropologie.

Neu ist die Ernsthaftigkeit, mit der über poetologische und anthropologische Konsequenzen aktueller neurobiologischer Theorien nachgedacht wird. Licht ins Dunkel des eigenen Gehirns zu bringen, heißt im Extremfall, einen Text zu produzieren, der auf der Basis der eigenen Gehirnaktivität entsteht und zugleich selbstreflexiv über die ihm zugrunde liegende Hirntätigkeit und die unhintergehbare, zugleich paradoxe Differenz zwischen dem Schreibenden und seinem Gehirn reflektiert. Für Grünbeins Gehirngedichte in der Schädelbasislektion ist dieses paradoxe Verhältnis, wie noch zu zeigen sein wird, entscheidend. Fioretos veranschaulicht es in einer Abwandlung des Märchens von Schneewittchen, in welcher der Text zum Zauberspiegel wird: „Spieglein, Spieglein vor mir, sag, wessen mein Gehirn sein mag!“"41 Anders ausgedrückt: Mit dem Wissen der Hirnforschung und ihrem Kontrast zur Selbstwahrnehmung der Person ist das Ich, das sich selbst im Schreibprozess zu erforschen versucht, sich zugleich darüber im Klaren, dass es immer auch ein ,Anderer‘, ein verschalteter Neuronenklumpen ist, dessen Areale größtenteils im Dunkeln liegen. Das ,Gehirn‘ wird dabei zum zugleich metaphorischen und realen Ort selbstreflexiver Überlegungen über die Vorgänge bei der Produktion von Literatur.

Die literarische Ausgestaltung subjektiver Erlebniswelten von Menschen, deren Selbst- und Weltwahrnehmung durch neurologische Störungen oder außergewöhnliche Gehirne verändert ist, ist eine weitere Form literarischer Kranionautik.

39 Parallel zu Fioretos' Konzept der Kranionautik heißt es bei Grünbein: Hirnbilder und Denkschriften, S. 23: „Der Trancezustand zeichnet sich dadurch aus, dass in ihm das vertraute Ich auf Wanderschaft durch die Auen der Anschauung geht. Reflektierend und Bilder sammelnd, streift es durch eine innere Flusslandschaft, während die Sinne offen für Umweltreize in erhöhte Alarmbereitschaft versetzt sind.“

40 Vgl. Jutta Müller-Tamm: WeltkörperInnenraum. Anmerkungen zur literarischen Anthropologie des Körperinneren. In: IASL 25.1 (2000). S. 95-133.

41 Fioretos: Mein schwarzer Schädel (Anm. 21), S. 10. Herv. C. W. 
Hier wird die „Selbstverständlichkeit, mit der die wissenschaftliche Perspektive sich selbst als allein legitime zur Erforschung des Menschen setzt“",22 auch auf dem Gebiet der Medizin hinterfragt. Fioretos präsentiert in seinem Essay Mein schwarzer Schädel ein ganzes literarisches Archiv subjektiver Innenperspektiven von Menschen in neurologischen Extremsituationen. Dazu gehört die literarische Erkundung der Innenperspektive eines am Locked-in-Syndrom Erkrankten aus dem Antikriegsfilm Johnny zieht in den Krieg nach dem Roman von Dalton Trumbo und die Ausgestaltung möglicher Erfahrungswelten von historisch überlieferten Janusköpfen, wie sie in Per Olov Enquists Roman Gestürzter Engel beschrieben werden. In allen Beispielen stellt sich die Literatur als alternative Hirnforschung dar: Dort, wo die Medizin die Krankheit, die Krankheitsgeschichte und den Nutzen für die Forschung sieht, leuchtet die Literatur die Krankengeschichte, die subjektive Innenperspektive der Betroffenen aus.

\section{Anthropologische Korrekturen - Imaginationsräume jenseits cartesianischer Dualismen}

Literarische Texte versammeln Wissen aus ganz unterschiedlichen Bereichen. ${ }^{43}$ Sie können in den Handlungen und Reden der Figuren, in Metaphern, Analogien und semantischen Verdichtungen inkommensurable Denkstile innerhalb einer Kultur zueinander ins Verhältnis setzen. ${ }^{44}$ Spannungen zwischen überlieferten Vorstellungen in einigen Wissensbereichen und neuen Erklärungsmustern in anderen können auf diese Weise sichtbar werden und auf Umbrüche innerhalb einer Kultur verweisen. Das erste Gedicht aus Durs Grünbeins Gedichtzyklus Schädelbasislektion konfrontiert, so meine These, gezielt das neue naturwissen-

42 Erik Porath: Literarische Kranionautik. Erzählweisen des Gehirns. In: Chiffre 2000 - Neue Paradigmen der Gegenwartsliteratur. Hrsg. von Corina Caduff und Ulrike Vedder. München: Fink, 2005. S. 43-58, hier S. 50. Das Konzept der ,literarischen Kranionautik' hat Erik Porath in seinem wegweisenden Aufsatz in Anlehnung an Fioretos entwickelt. Er fasst darunter alle Formen literarischer Auseinandersetzung mit dem Gehirn und der Hirnforschung. Wenngleich dem grundsätzlich zuzustimmen ist, liegt in diesem Kapitel (gleichfalls in Anlehnung an Fioretos' Essay) die Emphase auf der hirnphysiologisch informierten Darstellung subjektiver Innenperspektiven. 43 Vgl. Weigel, deren Ausführungen zum ,Schauplatz der Literatur' ich hier folge: Sigrid Weigel: Literatur als Voraussetzung der Kulturgeschichte. Schauplätze von Shakespeare bis Benjamin, München: Fink, 2004. S. 173.

44 Zum Gedankenverkehr innerhalb eines Denkstils (z. B. des naturwissenschaftlichen) und zwischen verschiedenen Denkstilen innerhalb einer Kultur vgl. Ludwig Fleck: Entstehung und Entwicklung einer wissenschaftlichen Tatsache. Frankfurt a. M.: Suhrkamp, 1980. Insbes. S. 129145. 
schaftliche Menschenbild mit dem älteren philosophisch-metaphysischen Bild vom Menschen. Es dreht sich um seine eigene Achse, um zwei sich ausschließende anthropologische Vorstellungen gleichzeitig präsent und in der Schwebe zu halten. Die anthropologische Korrektur besteht im Beitrag des Gedichts zur Gestaltung eines Menschenbilds, das die Spannung unterschiedlicher miteinander nicht kompatibler Teilperspektiven auf den Menschen aushält.

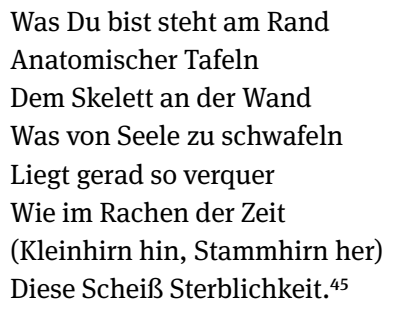

In der Forschungsliteratur ist dieses Gedicht wiederholt als Affirmation des naturwissenschaftlichen Blicks auf den Menschen gelesen worden. Es zeige, so Meike Adam, „das Ende der Metaphysik“ angesichts naturwissenschaftlicher Erkenntnisse. ${ }^{46}$ Sein Thema, seine Lektion, sei, so Wolfgang Riedel, die Bedeutung des „Körper[s] als letzte Instanz der Wirklichkeit und des Menschen“ und als Grundlage des von Grünbein vertretenen „physiologischen Humanismus“. Auch die Sterblichkeit bekomme, so Riedel weiter, einen anderen Stellenwert, wenn „keine unsterbliche Seele [...] mehr die Totgeweihtheit des Leibes aus[gleicht].“47

Die ersten vier Verszeilen präsentieren in der Tat mit kühl sezierendem Blick ein naturwissenschaftliches Menschenbild ohne metaphysische Dimension. Die zweite Hälfte des Gedichts widersetzt sich allerdings deutlich einer solchen Interpretation. Auf formaler Ebene kündigt sich dieser Widerstand im Klang der Kadenz an. Ihr Wandel vom melodisch leichten Rhythmus abwechselnder Kadenzen in den ersten vier Verszeilen zur rein männlichen Kadenz in der zweiten Hälfte

45 Grünbein: Schädelbasislektion. In: ders.: Schädelbasislektion, S. 11-15, hier S. 11.

46 Meike Adam: Augenblicks-Welten. Der radikale Konstruktivismus und Durs Grünbeins neurophysiologische Poetik. In: Der Schein der Dinge. Einführung in die Aesthetik. Hrsg. von Monika Fick. Tübingen: Attempo, 2002. S. 166-178, hier S. 175.

47 Wolfgang Riedel: Poetik der Präsenz. Idee der Dichtung bei Durs Grünbein. In: IASL 24.1 (1999). S. 82-105, hier S. 85-86. Stockhorst versteht das Gedicht als „ein implizites Nein zur wissenschaftlichen Erklärbarkeit des Menschen“, ohne diese Lesart jedoch zu begründen. Stefanie Stockhorst: Ästhetisierung der Anatomie. Medizinische und literarische Referenzräume in Durs Grünbeins Schädelbasislektionen. In: Schreiben am Schnittpunkt. Poesie und Wissen bei Durs Grünbein. Hrsg. von Kai Bremer, Fabian Lampert und Jörg Wesche. Freiburg: Rombach, 2007. S. 191-212, hier S. 208. 
des Gedichts führt, trotz der weiterhin konstanten Eingängigkeit regelmäßiger dreifüßiger Anapäste, zu einer Veränderung der Tonlage hin zu einer stärkeren Emphase - oder in den Worten Grünbeins zur Funktion der Kadenz: „[E]ben war es [das Wort, C. W.] noch flüchtig, und schon hält es traumschwer inne und sieht sich um in einer Welt, feindlich dem Doppelsinn.“48

Inhaltlich erschwert eine „gestörte Analogie“49 das Verständnis: „Dem Skelett an der Wand / Was von Seele zu schwafeln / Liegt gerad so verquer / Wie“ folgt kein einfacher Vergleich, sondern eine emotional aufgeladene (metrisch unregelmäßige) Metapher: „Wie im Rachen der Zeit / [...] / diese Scheiß Sterblichkeit“. Um die Metapher weiter aufzulösen, das tertium comparationis zu finden, ist das Körpergedächtnis des Rezipienten gefragt: Etwas, das quer im Rachen liegt, bietet einen Widerstand, lässt sich nicht schlucken, verdauen - ebenso wenig wie die Analogie selbst, die zum Innehalten und Nachdenken zwingt. Liegt die „Scheiß Sterblichkeit“ quer im „Rachen der Zeit“, so liegt das „Schwafeln“von der „Seele“ ebenso quer zum kühl zitierenden wissenschaftlichen Blick der ersten Verszeilen. Die Erinnerung an die eigene Sterblichkeit als unhintergehbares Faktum in der Tradition des memento mori ist eine Zentralaussage des Gedichts. Auf der Basis der übergeordneten Analogie folgt daraus, dass die Seele (oder das Reden über die Seele) ebenso wie die Sterblichkeit notwendig zum Menschen dazugehört. ${ }^{50}$ Das Gehirn taucht konsequenterweise in der zweiten Hälfte des Gedichts nur noch in einer Klammer (Kleinhirn hin, Stammhirn her) auf. ${ }^{51}$

48 Grünbein: Mein babylonisches Gehirn, S.26.

49 Ebd., S. 28.

50 Norbert Hummelt hat in seinem Nachwort zum Grünbein-Sammelband Limbische Akte am eindringlichsten auf die hochkomplexe rhetorische Figur im Zentrum des Gedichts hingewiesen und kommt zu einem ähnlichen Ergebnis: „das Wörtchen ,wie‘ [...] setzt das Schwafeln von der Seele im Angesicht des Knochenmanns in Relation zu einer anderen Aussage. Dass ,diese Scheiß Sterblichkeit ‘ . . . ], im Rachen der Zeit‘ liegt, dass also unser sterbliches Leben der alles verschlingenden Zeit in den Rachen geworfen wird, kann man kaum bezweifeln; wenn aber dem so ist und auch dieser Gedanke verquer liegt, so wie eine Gräte im Hals [...] dann ist [...] auch das Schwafeln von der Seele eine anthropologische Konstante, und alle Erkenntnisse der Hirnforschung helfen nicht darüber hinweg." Norbert Hummelt: Nachwort. In: Durs Grünbein: Limbische Akte. Gedichte. Ausgew. und mit einem Nachwort vers. von Norbert Hummelt. Stuttgart: Reclam, 2011. S. 354-355.

51 Aus der Perspektive der Evolutionsbiologie gehören Stammhirn und Kleinhirn zu den evolutionsgeschichtlich ältesten Bestandteilen des Gehirns. Insofern sie evolutionär ausgebildet vorliegen, könnte man sie als ,unsterblich` bezeichnen. Zum Stammhirneffekt in diesem Sinne bei Grünbein vgl. Waldemar Fromm: Die Literatur und das Gehirn. Zur Rezeption der Naturwissenschaften bei Botho Strauß und Durs Grünbein. Ein Vergleich. In: Kulturpoetik 9.2 (2009). S. 233251, insbes. S. 246. 
Vom Ende des Gedichts her gelesen erscheint das Schwafeln von der Seele im Zeitalter der Reduktion des Geistes auf das Gehirn als Widerstand gegen den Zeitgeist. Vom Anfang her gelesen bestätigt es hingegen das naturwissenschaftliche Menschenbild. Um sich widersprechende anthropologische Vorstellungen gleichzeitig präsent zu halten, dreht sich das Gedicht sozusagen um seine eigene Achse.

In seinem lyriktheoretischen Essay „Mein babylonisches Gehirn“ hat Grünbein eine solche „Drehung um die eigene Achse“ als einzige Grundbewegung charakterisiert, die dem Gedicht zur Verfügung steht, um „mit einem Minimum an Notiertem ein Maximum an Ausdruck zu erzielen““.52 Eine nachhaltige Wirkung entstehe im Gedicht dadurch, dass dieses durch metrische Barrieren, semantische Entfernungen zwischen Begriffen oder gestörte Analogien der formlos dahinfließenden Zeit „genügend Widerstände“ entgegensetze. Ihre Funktion ist die größtmögliche Verdichtung, Vernetzung, Spieglung von semantisch entfernten (beinahe unvereinbaren) Bildern und Begriffen auf engstem Raum. ${ }^{53}$

Das Gedicht baut, so lässt sich zusammenfassen, um eine komplex verschachtelte und metaphorisch erweiterte Analogie ein Spannungsverhältnis zwischen natur- und geisteswissenschaftlichem Menschenbild auf. Sie werden weder miteinander versöhnt, noch wird das eine zugunsten des anderen verworfen. Stattdessen werden beide Blickweisen über die verschachtelte Analogie unauflösbar ineinander verschränkt und gleichwertig in der Schwebe gehalten. Wichtig an dieser anthropologischen Korrektur ist, dass nicht die Entscheidung für oder gegen eine der beiden in sich kohärenten, aber inkompatiblen anthropologischen Positionen, sondern gerade die Gleichzeitigkeit beider als für das Selbstverständnis des Menschen im ausgehenden zwanzigsten Jahrhundert zentral gesetzt wird.

\section{Hirnforschung und Wissenschaftskritik in Durs Grünbeins Schädelbasislektion}

Grünbeins Gehirngedichte und seine poetologischen Reflexionen über die physiologischen und neurobiologischen Grundlagen von Dichtung gehören zu den

52 Diese und die folgenden Zit. aus Grünbein: Mein babylonisches Hirn, S. 27. Herv. C. W.

53 Über die Bedeutung der Zusammenfügung von semantisch Entferntem im Gedicht als Widerstand gegen Denkgewohnheiten und Möglichkeit einer grundlegenden Neuordnung der Konzepte nach der Lektüre vgl. auch Durs Grünbein: Katze und Mond. In: ders.: Galilei vermißt Dantes Hölle und bleibt an den Massen hängen. Frankfurt a. M.: Suhrkamp, 1996. S. 55-60. 
von der Literaturwissenschaft mit Vorliebe rezipierten Texten zum Thema ,Gegenwartsliteratur und Hirnforschung، $\cdot{ }^{54}$ Mit Emphase wird hier die Affinität der Dichtung zur naturwissenschaftlichen Anthropologie, die Rückkehr des Körpers (gemeint ist meistens das Gehirn) in den literarischen Text und der Anschluss der Literatur an die Neurowissenschaften hervorgehoben und zugleich der ästhetische Eigenwert der Literatur betont. ${ }^{55}$ Gern wird darauf verwiesen, dass Grünbein im Gedichtzyklus Schädelbasislektion die Seele ebenso wie die Autonomie des dichtenden Subjekts verabschiedet und an ihre Stelle das Gehirn als Dichter setzt. ${ }^{56}$ Selten wird jedoch gefragt, inwiefern Grünbein selbst die Ergebnisse und Möglichkeiten der Hirnforschung und die Theorien der Neurophilosophie in seiner Lyrik mit Blick auf ihren umfassenden Geltungs- und Deutungsanspruch kritisch reflektiert. ${ }^{57}$

54 Insbesondere die Gedichte in Schädelbasislektion (1991) und Falten und Fallen (1994) sowie die Essays Ameisenhafte Größe (1990), Den Körper zerbrechen (1995) und Mein babylonisches Gehirn (1995).

55 Vgl. u.a. Wolfgang Riedel: Poetik der Präsenz; Meike Adam: Augenblicks-Welten; Nicole Birtsch: Orientierungsversuche im Niemandsland zwischen Medizin und Poetik. Das Verhältnis von Körpergedächtnis und Poesie in den Texten von Durs Grünbein. In: Einschnitte. Identität in der Moderne. Hrsg. von Oliver Kohns und Martin Roussel. Würzburg: Königshausen \& Neumann, 2007. S. 103-115; Hinrich Ahrend: „Tanz zwischen sämtlichen Stühlen“. Poetik und Dichtung im lyrischen und essayistischen Werk Durs Grünbeins. Würzburg: Königshausen \& Neumann, 2007; Waldemar Fromm: Die Literatur und das Gehirn; Lothar van Laak: Die Bilder der Wissenschaft die Bilder der Literatur. Das interdisziplinäre Potential der Einbildungskraft im Blick von Eichendorff und Grünbein. In: Literaturwissenschaft interdisziplinär. Heidelberg: Synchron, 2010. S.131142; Anna Alissa Ertel: Körper, Gehirne, Gene. Lyrik und Literaturwissenschaft bei Ulrike Draesner und Durs Grünbein. Berlin: De Gruyter, 2011. Als Kritik an Grünbeins „Reduktion von Dichtung auf körperliche Prozesse“, die zugleich die Einschätzung von Grünbeins unkritischer Integration neurobiologischen Wissens in seine poetologischen und lyrischen Texte bestätigt, vgl. Achim Geisenhanslüke: ,Mensch ohne Großhirn‘. Durs Grünbein und das Ende der Utopien? In: Die eigene und die fremde Kultur. Exotismus und Tradition bei Durs Grünbein und Raoul Schrott. Tagungsprotokolle. Hrsg. von Dieter Burdorf. Iserlohn: Inst. für Kirche und Gesellschaft, 2004. S. 63-78, bes. S. 66-69.

56 Riedel: Poetik der Präsenz, S. 86-87; Adam: Augenblicks-Welten, S. 176-177; Ertel: Körper, Gehirne, Gene, S. 207-229, 235-242; Birtsch: Orientierungsversuche im Niemandsland.

57 Dürbecks informierte Darstellung von Grünbeins Kritik am physiologischen Reduktionismus in seinem Gedichtzyklus Mensch ohne Großhirn bietet hier eine erfreuliche Ausnahme. Vgl. Gabriele Dürbeck: „Wenn es stimmt, dass wir schwierige Tiere sind“. Anatomie und Anthropologie in Durs Grünbeins „Mensch ohne Großhirn“. In: Zeitschrift für Germanistik 19.1 (2009). S. 133145. In Ansätzen kritisch sieht auch Stefanie Stockhorst (Ästhetisierung der Anatomie) Grünbeins Stellung zu Hirnforschung. 
Richtungweisend für die Rezeption von Grünbeins Lyrik als „,neurophysiologische Poetik“ 58 war die Arbeit von Wolfgang Riedel. Von der Büchnerpreisrede ausgehend, hatte dieser Grünbeins „Bündnis mit den Naturwissenschaften“ 59 als dritten Weg angesichts der gegeben Alternativen politisch engagierter oder ästhetisch autonomer Dichtung dargestellt und dessen Poetik auf der Grundlage der neurokonstruktivistischen Wahrnehmungstheorie Gerhard Roths als eine „Poetik der Präsenz" beschrieben. ${ }^{60}$

Vor dem Hintergrund der Kritik von Thomas Fuchs am cartesianischen Erbe der kognitiven Neurowissenschaft und -philosophie wird deutlich, warum die konstruktivistische Neurophilosophie für die Literaturwissenschaft verlockend ist. Über Roths Definition der „Wirklichkeit“ als neuronales Konstrukt des Gehirns können bereits vorhandene ästhetische Theorien zur Produktion endogener Bilder in der Literatur mit der Produktion der Wirklichkeit durch das Gehirn gleichgesetzt und zu einer „Poetik der Präsenz“ (im Sinne einer Anwesenheit des Körpers und der Wirklichkeit im Text) umformuliert werden. Dass sich die Materialisierung der Einbildungskraft oder die Vorstellung des Gehirns als autonomer „Präsenz-Generator"61 der Beibehaltung eines alten Denkmusters verdankt, weiß auch Riedel. Er übersieht allerdings, dass mit dem alten Denkmuster auch die Trennung des autonomen übergeordneten Systems (Seele/Gehirn) vom (extrazerebralen) Körper beibehalten wird. Eine Poetik der Präsenz des Körpers lässt sich, nimmt man die Kritik von Fuchs ernst, mit Roths Neurophilosophie gerade nicht begründen.

Unbestreitbar bleibt, dass Grünbein sich für die Erkundung der neurobiologischen Grundlagen lyrischen Sprechens und kreativer Prozesse interessiert und den Menschen vom Körper her erkundet. Seine Aussage, dass „in der Neurologie die Poetik der Zukunft versteckt liege“, ist hier ebenso ernst zu nehmen wie die Überlegungen zur hirnphysiologischen Tiefenwirkung lyrischer Sprache. ${ }^{62}$ Es darf jedoch nicht übergangen werden, dass Grünbein selbst, wenn er vom Körper redet, nicht immer das Gehirn meint, ${ }^{63}$ und wenn er vom Gehirn redet, öfter die subkortikalen Hirnareale (Stammhirn, Kleinhirn, Zwischenhirn) fokussiert als

58 So der Untertitel von Adam: Augenblicks-Welten.

59 Riedel: Poetik der Präsenz, S. 83.

60 Ebd., S. 90-98.

61 Ebd., S. 96.

62 Durs Grünbein: Mein babylonisches Hirn, S. 21.

63 Vgl. Durs Grünbein: Reflex und Exegese. In: ders. Galilei vermißt Dantes Hölle, S. 62: „Bloßes Lesen genügt nicht, hat nie genügt. Entweder spricht der Körper, über die weißen Seiten gebeugt, mit, oder das Gedicht geht spurlos an ihm vorbei direkt ins Leere [...] ohne Spannungsaufbau kein Magnetfeld.“ 
das Großhirn (Neokortex, Assoziationskortex), dem Sitz höherer kognitiver Leistungen einschließlich endogener Bildproduktionen. ${ }^{64}$

Daher möchte ich anhand einer Zusammenschau zweier Gedichte aus dem Gedichtband Schädelbasislektion mit seinerzeit aktuellem Wissen der Psychopharmakologie und der Neurotransmitterforschung zeigen, dass diese Gedichte sich für einen ganz anderen Bereich der Hirnforschung interessieren als den der kognitiven Neurowissenschaften. Ihr Interesse an den subkortikalen Hirnarealen, so die These, speist sich aus der Frage sowohl nach deren Bedeutung für das Dichten und Denken als auch nach den Möglichkeiten, mit dem neurochemischen Wissen „modellierend in geistig-seelische Funktionen ein[zu]greifen.“65 Auf dieser Ebene wird die zeitgenössische Hirnforschung zu Beginn der ,Dekade des Gehirns ' in den Gedichten zugleich interessiert rezipiert und kritisch reflektiert.

\title{
Das Gehirn und seine Drogen
}

\author{
Ohne Drogen läuft nichts \\ Hier im Irrgang der Zeichen \\ Wo Du umkommst gesichts- \\ Los in blinden Vergleichen \\ Träumend... Rate für Rate \\ Von den Bildern beäugt. \\ Wer ist Herr der Opiate \\ Die das Hirn selbst erzeugt? ${ }^{66}$
}

Grundlage dieses vierten Gedichts aus dem Zyklus Schädelbasislektion ist die bereits angesprochene paradoxe Differenz zwischen dem Schreibenden und seinem Gehirn. Sie ist hier eingebettet in eine selbstreflexive Lyrik, die über die Bedingungen ihrer schöpferischen Kreativität reflektiert, indem sie sich dem Ort ihrer Entstehung, dem Gehirn zuwendet. Das Gehirn erscheint dabei für den Sprecher des Gedichts als ein nicht ungefährlicher Ort: Der gebrochene Reim auf „Nichts“ (,gesichts-Los“) deutet die Auflösung des Subjekts an, in einem Gehirn, dessen Fremdheit sprachlich-kognitiv nicht zu fassen ist - die Vergleiche sind blind, sie ermöglichen keine Erkenntnis.

64 Nicht immer beachtet wurde dabei zudem, dass sich seit Anfang der 1990er Jahre die Hirnforschung und mit ihr die Neurophilosophie rasant entwickelt hat. Eine Rezeption vom Zusammenhang zwischen Gedicht und Gehirn in Schädelbasislektion muss demnach wissensgeschichtlich vom Stand der Forschung vor 1991 ausgehen.

65 Solomon H. Snyder: Chemie der Psyche. Drogenwirkungen im Gehirn [Drugs in the Brain, 1986]. Heidelberg: Spektrum der Wissenschaft, 1988. S. 214.

66 Grünbein: Schädelbasislektion, S. 14. 
Ein Gedicht über die Dichtung also, das zu dem Ergebnis kommt, dass das Gehirn und nicht das Subjekt dichtet? ${ }^{67}$ Es leuchtet auf den ersten Blick ein, zumal die endogenen Bilder deutlich genug als Produktion des Gehirns gekennzeichnet werden. Das Bewusstsein ist ihnen passiv ausgeliefert, keineswegs Herr über die Bildproduktion des Gehirns. Die Frage des Gedichts lautet allerdings nicht: „Wer dichtet?“, sondern: „Wer ist Herr der Opiate / Die das Hirn selbst erzeugt?“ Gefragt wird nach dem genaueren Produktionsort, insbesondere nach der die Produktion regulierenden Instanz endogener Opioide. Diese Frage war in der Neurobiologie und der Neuropharmakologie Ende der 1980er Jahre höchst aktuell und keineswegs vollständig geklärt. Deutlich wurde damals, dass ihre Beantwortung nicht nur die Autonomie des Subjekts, sondern auch die These vom autonomen, den anderen Hirnarealen übergeordneten Neokortex infrage stellte.

Solomon H. Snyder, einer der ,Entdecker' dieser Wirkstoffe und ihrer Rezeptoren, hat das damalige Wissen populärwissenschaftlich aufgearbeitet. Seine Monographie zur Chemie der Psyche. Drogenwirkungen im Gehirn erschien 1988 bei Spektrum der Wissenschaft in der ersten, bereits ein Jahr später in der zweiten Auflage. ${ }^{68}$ Neben einer Einführung in die elektrochemische Erregungsübertragung an den Nervenenden und in die Bedeutung einzelner Hirnareale gibt das Buch einen klar verständlichen Überblick über die Hypothesen zur Wirkungsweise von Psychopharmaka, körpereigenen Opioiden und anderen Neurotransmittern.

67 So wird das Gedicht durchgängig interpretiert. Vgl. Riedel: Poetik der Präsenz, S. 87: „Diese zerebrale Chemie hat keinen ,Herrn' über sich namens Vernunft oder Geist; sie selbst kocht die ,Bilder aus, von denen das Bewußtsein des Dichters sich umgeben sieht. Wer dichtet also? Die Physis selbst, das ,Hirn'. Noch hier, im scheinbar leibfern ideierenden poetischen Prozeß, ist der Körper prima causa, letzte Instanz“. Vgl. auch Ertel: Körper, Gehirne, Gene, S. 236-238; AnneRose Meyer: Physiologie und Poesie. Zu Körperdarstellungen in der Lyrik von Ulrike Draesner, Durs Grünbein und Thomas Kling. In: Gegenwartsliteratur. Ein germanistisches Jahrbuch 1 (2002). S. 107-133, bes. S. 120. Etwas vorsichtiger formuliert es Fromm: Die Literatur und das Gehirn, S. 245: „Gefragt wird nach dem lyrischen Ich unter den Bedingungen seiner Körperlichkeit und der Intensität des Dichtens [...]. Thema ist das Zeichenproduzierende Gehirn als Entstehungsort der Dichtung. Bewusstsein fragt nach dem Status seiner materiellen Voraussetzungen, seiner ,Regulierung، von diesen Voraussetzungen aus.“

68 Seit der Veröffentlichung von Grünbeins Schädelbasislektion im Jahre 1991 hat sich das Wissen über Wirkungsweisen endogener Opiate und spezifischer, die Grenzen zwischen den einzelnen Hirnarealen überschreitenden Nervenbahnen vervielfältigt. Der folgende Überblick präsentiert das dem Gedichtzyklus zeitgenössische Wissen. Ich beziehe mich größtenteils auf Snyders Darstellungen. Hinsichtlich des Entstehungsorts der Opioide habe ich die an ein breiteres Fachpublikum gerichtete Überblicksdarstellung der Forschungsfragen und Ergebnisse zur Neurochemie des Gehirns hinzugezogen. Jüngere Forschungsaufsätze werden nur dort ergänzend eingebracht, wo sie in Ansätzen einen wissensgeschichtlichen Überblick über die Erforschung endogener Opioide bieten. 
Bei den vom Gehirn selbst erzeugten Opiaten handelt es sich um endogene Opioide, die natürlichen Liganden der Opioidrezeptoren mit opium- oder morphinähnlicher Wirkung. $\mathrm{Zu}$ diesen Substanzen gehören verschiedene Endorphine, Enkephaline und Dynorphine. Sie werden u. a. in Stresssituationen ausgeschüttet und wirken schmerzstillend, euphorisierend und angstlösend. Die höchste Opioidrezeptordichte fand man laut Snyder im Zwischenhirn (im Hypothalamus) und in der Amygdala, einem Teil des limbischen Systems, das eine bedeutende Rolle bei der Entstehung von Gefühlen und Stimmungen spielt. Durch die enge neuronale Verknüpfung des limbischen Systems mit dem Hypothalamus und der Großhirnrinde wirken Emotionen sowohl auf den Körper (etwa durch die Ausschüttung von Hormonen über den Hypothalamus) als auch auf die Denk- und Gedächtnisprozesse. ${ }^{69}$

Bereits die Antike kannte die Wirkung des Opiums und setzte sie medizinisch ein. Die Opiatrezeptoren an postsynaptischen Membranen der Neuronen wurden jedoch erst 1973 identifiziert. $^{70} 1975$ wurde das Enkephalin als erstes körpereigenes Opioid aus Hirnextrakten toter Ratten isoliert. ${ }^{71} \mathrm{Im}$ selben Jahr wurde nach derselben Methode, die zur Identifizierung der Opioidrezeptoren geführt hatte, auch der Dopaminrezeptor gefunden, der die Erkundung der für den Emotionshaushalt wichtigen dopaminergen Nervenbahnen einleitete und bei der Entwicklung von Neuroleptika zur Behandlung schizophrener Denkstörungen eine zentrale Rolle spielte. ${ }^{72}$ Zehn Jahre später, 1985, gelang es mit dem bildgebenden Verfahren der Positronen-Emissions-Tomographie (PET) erstmalig in vivo, also im lebenden menschlichen Gehirn, die durch endogene Opioide in den verschiedenen, insbesondere subkortikalen Hirnregionen ausgelöste neuronale Aktivität sichtbar zu machen. ${ }^{73}$ Die Möglichkeiten der PET zur quantitativen Erfassung und visuellen Kartierung der durch Opioide veränderten Neuronenaktivität in spezifischen kortikalen und subkortikalen Hirnarealen haben seitdem das Wissen um Vorkommen und Wirkungsweisen nicht nur der Opioide, sondern auch der Neurotransmitter insgesamt revolutioniert. ${ }^{74}$

69 Vgl. Snyder: Chemie der Psyche, S. 47-67. Zur Bedeutung des limbischen Systems vgl. S. 54-58. 70 Ebd., S. 48.

71 Ebd., S. 60-65.

72 Ebd., S. 84-93.

73 Vgl. Gjermund Henriksen und Frode Willoch: Imaging of opioid receptors in the central nervous system. In: Brain:Journal of Neurology 131 (Mai 2008). S. 1171-1196, bes. S. 1178.

74 Vgl. Terry Joney und Eugenii Rabiner: The development, past achievements, and future directions of brain PET. In: Journal of Cerebral Blood Flow \& Metabolism 32 (Juli 2012). S. 1426-1454, bes. S. 1427-1428. 
Diese kurze Entdeckungsgeschichte der endogenen Opioide zeigt, dass die Frage des Gedichts nach dem ,Herrn' der Opiate einen in den 1980er Jahren hochaktuellen Bereich der Neurochemie und Psychopharmakologie anschneidet. Deutlich wird ihre damalige Bedeutung für die Erkundung der Organisation und Arbeitsweise des Gehirns und die Erforschung des Bewusstseins. Bereits in den Dekaden vor der Aufwertung der Emotionen in den kognitiven Neurowissenschaften der 1990er Jahre hatte die Beforschung der Wirkungsweise von endogenen und exogenen Drogen wie auch Psychopharmaka die Aufmerksamkeit auf die subkortikalen Hirnregionen sowie auf deren Bedeutung auch für höhere kognitive Leistungen gelenkt. ${ }^{75}$ Hauptproduktionsort endogener Opioide ist demnach das Zwischenhirn und dort größtenteils der Hypothalamus, wo sie als Zerlegungsprodukte größerer Proteine entstehen. ${ }^{76}$ Da der Hypothalamus das zentrale Steuerungssystem des autonomen (vegetativen) Nervensystems ist, liegt es nahe, ihm eine Funktion auch bei der Regulation der Opioidproduktion zuzugestehen.

Beantwortet man die Frage des Gedichts in diese Richtung, wird erkennbar, dass im Gedicht die Bedeutung subkortikaler, bewusst kaum steuerbarer Prozesse für die Herstellung eines Zustands künstlerischer Kreativität reflektiert wird. Eine der Lektion des Gedichtzyklus Schädelbasislektion wäre demnach, dass das Großhirn als Ort höherer kognitiver Leistungen gerade nicht autonom, nicht Herr der Opiate (und damit auch nicht im Freud'schen Sinne Herr im Hause) ist. Seine Funktion und mit ihr auch die endogene Bildproduktion wird von den subkortikalen Hirnarealen mitbestimmt. Die Verszeilen „Träumend... Rate für Rate / Von den Bildern beäugt“ beziehen sich direkt auf die Wirkung der in diesen Hirnarealen produzierten Opioide: „Der euphorische Zustand, den Opiate auslösen, ist ein eher passives, traumartiges Geschehen. "77 Im Zentrum des Gedichts steht demnach nicht die Reflexion darüber, dass die „zerebrale Chemie [...] keinen ,Herrn' [...] namens Vernunft oder Geist“" über sich hat und stattdessen „das Hirn“ dichtet. ${ }^{78}$ Vielmehr bildet das neurobiologische Wissen um die Wirkungsweise endogener Opioide zusammen mit den damals noch offenen Fragen nach der körperinternen Regulation den Ausgangspunkt der Überlegun-

75 In den kognitiven Neurowissenschaften wurde die Bedeutung des das limbischen Systems für das Denken erst im Zusammenhang mit der Renaissance der Gefühle in den 1990er Jahre erkannt. Die Standardwerke von Gerhard Roth (Das Gehirn und seine Wirklichkeit) und António Damásio (Descartes' Irrtum; Ich fühle also bin ich) erschienen ab 1994, also später als Grünbeins Schädelbasislektion (1991).

76 Rudolf Nieuwenhuys: Chemoarchitecture of the Brain. Berlin: Springer, 1985. S. 94-108.

77 Snyder: Chemie der Psyche, S. 209.

78 Riedel: Poetik der Präsenz, S. 87; und mit ihm u. a. Ertel: Körper, Gehirne, Gene, S. 236-238. 
gen zur innerphysiologischen Grundlage lyrischer Produktivität jenseits des Einflussbereichs der Großhirnrinde. Die Verunsicherungen des Selbstbewusstseins durch die Hirnforschung liegen nicht allein darin, dass höhere kognitive Fähigkeiten als Ergebnisse neuronaler Prozesse gefasst werden. Einschneidender noch erscheint die Möglichkeit, dass die neuronale Informationsverarbeitung selbst durch neurochemische Prozesse modifiziert wird, die weder das Großhirn noch das Subjekt kontrollieren können.

\section{Perspektivverschiebungen: Vom Neokortex zum Zwischenhirn}

Grünbeins „Ode an das Dienzephalon (nach W. H. Auden nach A. T. W. Simeons)“ aus dem Gedichtband Schädelbasislektion konzentriert sich explizit auf das Zwischenhirn. ${ }^{79}$ Ging es in dem vorausgehenden Gedicht um eine interessierte Rezeption eines spezifischen Teilgebiets der Hirnforschung mit Blick auf die Bedeutung der Neurotransmitterforschung sowohl für das anthropologische Selbstverständnis als auch für neurobiologisch informierte Dichtungstheorien, so findet sich hier - neben einer Integration neurobiologischen Wissens um die Bedeutung subkortikaler Hirnareale - auch eine kritische Reflexion der anthropologischen Konsequenzen neuropharmakologischer Forschung.

Bereits im Titel von Grünbeins „Ode“ wird eine historisch-spezifische Theorie zur Wirkungsweise des Zwischenhirns mit verschiedenen Facetten des spezifisch literarischen Wissens um Gedichtformen und ihre Herkunft sowie um andere literarische Texte zusammengeführt. Grünbein hat für den griechischen Begriff ,Dienzephalon' ganz passend ein antikes griechisches Versmaß - das der sapphischen Ode - gewählt. Sie wurde nach der antiken Lyrikerin Sappho benannt, die in dieser Odenform Götterhymnen und Liebeslieder verfasste. Folgt man dem Hinweis auf Auden, so stellt sich schnell heraus, dass dieser ein Vierteljahrhundert zuvor (1972) ebenfalls eine sapphische Ode an das Dienzephalon veröffentlicht hatte: „Ode to the Diencephalon (after A. T. W. Simeons)““.80 Albert Simeons wiederum war Endokrinologe und beschäftigte sich vor allem mit Hormonen, deren Produktion vom Hypothalamus aus, einem Teil des Zwischenhirns, gesteu-

79 Grünbein: Ode an das Dienzephalon (nach W. H. Auden nach A. T. W. Simeons). In: ders.: Schädelbasislektion, S. 133.

80 Wystan H. Auden: Ode to the Diencephalon (after A.T. W. Simeons). In: ders.: Collected Poems. Hrsg. von Edward Mendelson. New York: Vintage International, 1991. S. 878. 
ert wird. ${ }^{81}$ Gegen Ende seiner Karriere interessierte sich Simeons im Zusammenhang mit psychosomatischen Erkrankungen für die Interaktion zwischen Kortex und Zwischenhirn. In diesem Kontext erschien 1960 seine Monographie zu den Ursachen psychosomatischer Störungen: Man's Presumptuous Brain. An Evolutionary Interpretation of Psychosomatic Disease. In dieser Abhandlung werden die Grenzen des Bekannten bewusst zugunsten einer übergreifenden Hypothese menschlichen Verhaltens im Allgemeinen und psychosomatischer Störungen im Besonderen überschritten. Die Evolutionsbiologie bildet dabei das übergeordnete Narrativ, in das zeitgenössisches Wissen über Funktionsweise und Aufgaben des Dienzelphalon eingetragen wird. ${ }^{82}$

Der Bezug von Grünbeins Ode aus dem Jahre 1991 zur Theorie Simeons' und zum Gedicht Audens ist von der Forschung mehrfach thematisiert worden. ${ }^{83} \mathrm{Nicht}$ berücksichtigt wurde dabei die zeitliche Differenz zu den Prätexten von Simeons (1960) und Auden (1972) sowie die dazwischen liegende Verschiebung der Forschungsinteressen im Zusammenhang mit der Erforschung der Wirkungsweise von Psychopharmaka und endogenen Neurotransmittern. Die wissensgeschichtliche und wissenschaftskritische Dimension des Gedichts erschließt sich jedoch erst in einem intertextuellen Vergleich beider Oden, der zugleich auch den jeweils zeitgenössischen Forschungsstand zum Zwischenhirn miteinbezieht.

Nach Simeons spielte das Dienzephalon vor allem für die Vorbereitung des Körpers auf Flucht oder Angriff, also für zwei zumindest im Tierreich überlebenswichtige Reaktionen auf Umweltereignisse, eine zentrale Rolle. Bei den höher entwickelten Säugetieren ist, so die These, die Zusammenarbeit zwischen Kortex und Dienzephalon für diese Aufgabe gut eingespielt: Der Kortex registriere und verarbeite die Sinnesdaten und leite nur dann Information an das Dienzephalon weiter, wenn eine überlebenswichtige Handlung ausgelöst werden soll. Alle anderen Informationen aus der Umwelt würden nicht an das Dienzephalon weitergeleitet, sondern durch einen Zensor blockiert. Das Problem des zivilisierten Menschen besteht nach Simeons darin, dass die ,alte Zensurierung der Sinnesbotschaften nicht auf die neuen, von der Kultur geschaffenen Bedingungen“ hin abgeändert wurde. ${ }^{84}$ Der Zensor leite nach wie vor die Gefahren von einst vom Kortex an das

81 Zum Wissensstand der 1950er Jahre vgl. Wolfgang Bargmann: Das Zwischenhirn-Hypophysensystem. Berlin: Springer, 1954; W. R. Hess: Das Zwischenhirn. Syndrome, Lokalisationen, Funktionen. Basel: Schwabe, 1952, bes. S. 70-87.

82 Vgl. die deutsche Ausgabe: Albert T. W. Simeons: Das tyrannische Gehirn als Ursache seelisch bedingter Leiden. Rüschlikon-Zürich: A. Müller, 1962.

83 Vgl. Stockhorst: Ästhetisierung der Anatomie, S. 198-203; Ertel: Körper, Gehirne, Gene, S. 167177.

84 Simeons: Das tyrannische Gehirn, S. 60-66, bes. S. 65. 
Dienzephalon weiter. Das Dienzephalon reagiere wie immer mit automatischen Reflexen, die nun aber der Situation unangemessen sind. Erklärt wird diese fehlende Adaption mit dem Grundargumentationsmuster der Evolutionsbiologie: erstens mit der Geschwindigkeit der kulturellen Entwicklung im Vergleich zu den viel langsamer verlaufenden evolutionären Prozessen; zweitens mit der Spezialisierung der Hirnareale und Zensurmechanismen, die nicht aufgrund neuer Umweltbedingungen umprogrammiert werden können.

Audens Ode knüpft an Simeons' These von evolutionsbiologisch bedingten Kommunikationsstörungen zwischen Dienzephalon und Kortex durch die mangelhafte Anpassung des Gehirns an die moderne Zivilisation an, gibt nun aber dem Dienzephalon selbst, nicht der Evolution die Schuld. In der ersten Strophe als ungeschliffen beschimpft, wird dem Dienzephalon vorgeworfen, dass es noch immer nicht gelernt habe, die Botschaften des Kortex richtig zu beurteilen:

How can you be so uncouth? After sharing the same skull for all these millennia, surely you should have discovered the cortical $I$ is a compulsive liar.

Hinsichtlich der durch grundlegende Leistungen der Zivilisation veränderten Umweltbedingungen ist das Dienzephalon hoffnungslos ignorant. Auch gesellschaftliche Strukturen, die an die Stelle des darwinistischen Überlebenskampfes Recht und Gesetz stellen, sind ihm unbekannt. Es reagiert nach wie vor mit den eingespielten Reaktionsmustern von Flucht und Angriff:

He has never learned you, it seems, about fig-leaves

or fire or ploughshares or vines or policemen,

that bolting or cringing can seldom earth a

citizen's problems?

In der entscheidenden Wende des Gedichts, in der vierten und letzten Strophe, wird schließlich deutlich, worauf Audens Kritik am Dienzephalon hinaus will und warum es dennoch eine Ode ist, als Anrufung einer höheren Instanz, sich endlich einzuschalten:

When you could really help us, you don't. If only, whenever the trumpet cries men to battle, you would flash to their muscles the urgent order ACUTE LUMBAGO! ${ }^{85}$

85 Auden: Ode to the Diencephalon. Herv. im Orig. 
Auden greift, so lässt sich zusammenfassen, Simeons' These von der mangelnden Adaption des menschlichen Gehirns an die Bedingungen der Zivilisation auf und veranschaulicht sie anhand von Beispielen. Mit der Verschiebung der Verantwortung an das Dienzephalon klingt zudem eine allgemeine Vernunftkritik an, die mit einer Wendung ins Politische einhergeht. ${ }^{86}$ Der Kortex erhält im Gedicht lediglich eine Nebenrolle. Den höheren, dem Menschen spezifischen geistigen Eigenschaften wird eine Lösung zentraler Probleme der Zivilisation gar nicht erst zugetraut allenfalls archaische Instinkte könnten, wenn das Dienzephalon seiner eigentlichen Aufgabe nachginge, dem gegenseitigen Morden Einhalt gebieten. Das Gedicht Audens entpuppt sich damit als Antikriegsgedicht. Simeons' Theorie wird in ihrer literarischen Umsetzung weniger reflektiert als vielmehr für eine politische Aussage funktionalisiert.

Neben der Übernahme der Form und des Titels korrespondiert Grünbeins „Ode an das Dienzephalon“ auf zweierlei Weise erkennbar mit Audens Gedicht. In Grünbeins Ode gibt es ebenso wenig wie in der Audens ein lyrisches, also ein erlebendes Ich, das sich, wie in anderen Oden üblich, zu dem (oder der) von ihm Angesungenen in Beziehung setzt. Das ,Ich' tritt in seiner grammatikalischen Funktion als Verweis auf ein artikulierendes Ich des Gedichts gar nicht in Erscheinung. Wer genau von welchem Ort aus spricht, ist nicht ganz klar. Dennoch taucht das ,Ich` als Wort, bei Grünbein als Akkusativ-Objekt, bei Auden als „cortical I“ auf. Es erscheint als das Andere, Fremde, Bedrohliche, als das kortikale, rationale Ich, das Cogito des René Descartes. Als solches nimmt es in beiden Oden, die sich dezidiert an das Dienzephalon richten, die Position des Dritten, aus dem Gespräch Ausgeschlossenen ein.

Weitere Bezüge zu Audens Ode, vor allem in der dritten und vierten Strophe, dienen der Herausbildung eines im Vergleich zu Auden gänzlich anderen Blicks auf das Dienzephalon und seiner Zuständigkeiten im Reich des Gehirns. Hatte Auden dasselbe als ungeschliffen, unwissend und uninformiert über die Vorgänge sowohl im Kortex als auch in der Welt beschimpft, so erscheint Grünbeins Dienzephalon ganz im Gegenteil als die überlegene, alles überblickende Kontrollinstanz. Es beherrscht nicht nur die grundlegenden Errungenschaften der Zivilisation, sondern auch höhere Kulturleistungen: „Alles im Griff, Flugkörper, Sprachen und Religionen“. ${ }^{87}$ Über die Tätigkeiten des Neokortex scheint es bestens informiert, nimmt jedoch dessen Höhenflüge und Aufstiegsambitionen nicht wirklich ernst:

86 Zum selben Ergebnis von Audens Ode kommt Stockhorst: Ästhetisierung der Anatomie, S. 201. Grünbeins Ode wird von Stockhorst (ebd., S. 203-204) anders als hier in den Kontext des Behaviorismus eingeordnet.

87 Grünbein: Ode. Hiernach auch das Folgende. Herv. C. W. 
Nichts von dem was sich im Neuronalen Netz fing War dir wirklich Ernst. Selten stand mehr auf dem Programm Als Betrug, psychische Tricks oder Schlüsse wie dieses Cogito ergo...

Das cartesianische cogito ergo sum, ein für die Selbstdefinition des rationalistischen Menschen und die Begründung seiner Erkenntnisfähigkeit zentraler Satz, erscheint aus der dem Dienzephalon unterstellten Perspektive als eine für das Überleben unwesentliche, nicht ernst zu nehmende Spielerei. Mit dieser literarisch überzeichneten Aufwertung des Zwischenhirns zur zentralen Instanz des Gehirns zeichnet die Ode unter Verweis auf Simeons und Auden die Verschiebungen in den Forschungsfragen an das Gehirn seit den 1970er Jahren nach.

Bereits in der ersten Strophe wird das Dienzephalon als das von der Hirnforschung lang übersehene, bisher ungreifbare (und damit auch unangreifbare, nicht durch gezielte Eingriffe manipulierbare), schließlich aber dennoch entdeckte Zentrum des Gehirns präsentiert: „Hier also hältst du, Black Box, dich versteckt. [...] / Lange warst du ungreifbar, nun bist du dir selbst / Häßlich der Nächste.“

Ebenso wie im oben besprochenen vierten Gedicht des Gedichtzyklus Schädelbasislektion führt auch hier eine Frage zu dem Problem der jüngeren Forschung. Sie findet sich im Zentrum des Gedichts, am Ausgang der zweiten Strophe: „Eingeklemmt zwischen Logos und feeling ... fragt sich / Wer hält hier wen fest?“ Hirnanatomisch gesehen („Klinisch entblößt, auf Karten verzeichnet“) liegt das Zwischenhirn mit seinen zentralen Kernen des Thalamus und des Hypothalamus zwischen dem Kortex („Logos“) und dem limbischen System („feeling“). Der Adoneus dieser zweiten Strophe fragt nach Hierarchie, nach den Machtverhältnissen innerhalb des Gehirns.

Aus der Perspektive der Neurotransmitter- und Psychopharmakaforschung der 1980er Jahre erscheint die Richtung der Antwort klar: Wohl ist der Kortex das Zentrum des Bewusstseins und menschlicher Kognition, dennoch ist auch der Kortex in seiner Funktionsfähigkeit auf das Dienzephalon und andere subkortikale Areale angewiesen. Als Steuerungszentrale des zentralen Nervensystems, des inneren Milieus, sowie als Ort der Produktion bzw. Regulation von Opioiden und Hormonen übt der Hypothalamus einen erheblichen Einfluss auf die Funktionsweise des Großhirns aus. Als ,Tor zum Bewusstsein' dient der Thalamus als Schaltstelle sensorischer Daten. Simeons' Darstellung der Informationsübermittlung zwischen Kortex und Dienzephalon hat sich als falsch herausgestellt: Die Informationsverarbeitung der Daten von den Sinnesorganen verläuft gerade umgekehrt, von den Sinnesorganen über ein Kerngebiet des Thalamus zum Kortex. Automatische Angstreaktionen erfolgen über eine direkte Verbindung zwischen 
dem Thalamus und dem limbischen System ohne Beteiligung des Kortex. Auf dieser Ebene haben sich die Forschungsthesen über die Machtverhältnisse im Zusammenspiel der Hirnareale (,Wer hält hier wen fest?“) verschoben: Man geht nun davon aus, dass nicht der Kortex, sondern das Dienzephalon darüber entscheidet, welche Sinnesdaten in welche Hirnareale weitergeleitet, welche Drogen und Hormone wann ausgeschüttet werden. Die Großhirnrinde verliert ihre Position als eine den anderen Hirnarealen übergeordnete Instanz in einem klar hierarchischen Top-Down-Modell.

Neben dieser wissensgeschichtlichen Dimension enthält Grünbeins Ode eine wissenschaftskritische Dimension und kann, so möchte ich abschließend zeigen, auch als kritischer Kommentar zu neuropharmakologischen Interessen am Gehirn gelesen werden. Die letzte Strophe des Gedichts (deren Stoßrichtung in der oben zitierten zweiten Hälfte der ersten Strophe bereits angedeutet wird) verweist darauf, dass dem Dienzephalon gerade durch seine wissenschaftliche Entdeckung ein Verlust seiner Machtposition droht:

\section{Alles im Griff, Flugkörper, Sprachen und Religionen, Hast Du nur eins unterschätzt, dieses Ich. Besser Es läge noch immer vor seinem Schlag aus der Art Glücklich im Koma.}

Will man sich mit der evolutionsbiologischen Feststellung nicht begnügen, das kortikale Ich werde hier als evolutionäre Fehlentwicklung präsentiert ${ }^{88}$, lohnt ein Blick auf die Strophenausgänge: „Häßlich der Nächste“, „Wer hält hier wen fest?“, „Cogito ergo“, „Glücklich im Koma“.

Auf dieser Ebene des Gedichts dominiert überraschenderweise das kortikale Ich: Es dominiert deutlich in den Adonei der beiden letzten Strophen, im cogito ergo und im Ich, das eben nicht glücklich im Koma liegt. Doch auch der erste Adoneus bezieht sich auf das Dienzephalon nach seiner wissenschaftlichen Entdeckung, an der unweigerlich das kortikale Ich, die cartesianische Ratio einiger Forscher beteiligt war. Bleibt der einzige nicht direkt zuzuordnende Adoneus, der selbst das Problem der Zuordnung reflektiert, die Frage: „Wer hält hier wen fest?“

Im Hinblick auf die Strophenausgänge wird deutlich, dass die Machtverhältnisse durch den wissenschaftlichen Blick auf das Gehirn ins Wanken geraten sind. Unterschätzt hat das Dienzephalon das kortikale Ich offensichtlich in seiner wissenschaftlichen Neugier und den für das Dienzephalon selbst aus diesem Forschungsdrang entstehenden Konsequenzen: Die überlegene Einstellung des Dienzephalons zum kortikalen Ich (,Nichts von dem was sich im Neuronalen Netz

88 Stockhorst: Ästhetisierung der Anatomie, S. 203. 
fing / War dir wirklich Ernst“) erweist sich im Nachhinein als Fehleinschätzung. Nach seiner wissenschaftlichen Entdeckung ist das Dienzephalon weder ,ungreifbar" noch unangreifbar, sondern durchaus von außen mit den entsprechenden Psychopharmaka manipulierbar. Die sapphische Ode an das Dienzephalon ist eine Hymne an einen von der Forschung entmachteten Gott. Das lyrische Ich stellt sich dabei eindeutig auf die Seite des Dienzephalons - das kortikale Ich erscheint als der aus dem Gespräch ausgeschlossene Dritte, der Fremde. Oder mit den Worten Grünbeins:

Während das kognitive Ich aus der Weltbeherrschung entsteht, indem es das Reale erfasst, berechnet, vermisst und gestaltet, konzentriert das lyrische sich ganz auf die Anschauung. Es lehnt sich zurück und geht den Tag- und Nachtträumen nach, den Phantasien, Nachbildern, Erinnerungen. Eine seiner Bestimmungen lautet: Es ist der Statthalter der Stimme, wobei Stimme sich als Logos der Rede erweist, die sinnstiftende Elementarkraft im Vers. Das lyrische Ich wäre demnach jene Steuerungsinstanz, die den Geist in die Gedichtzeilen schmuggelt, während die Sinne beschäftigt sind mit der Wahrnehmung der Welt. ${ }^{89}$

\section{Literatur}

Adam, Meike: Augenblicks-Welten. Der radikale Konstruktivismus und Durs Grünbeins neurophysiologische Poetik. In: Der Schein der Dinge. Einführung in die Aesthetik. Hrsg. von Monika Fick. Tübingen: Attempo, 2002. S. 166-178.

Ahrend, Hinrich: „Tanz zwischen sämtlichen Stühlen“. Poetik und Dichtung im lyrischen und essayistischen Werk Durs Grünbeins. Würzburg: Königshausen \& Neumann, 2007.

Auden, Wystan H.: Ode to the Diencephalon (after A. T. W. Simeons). In: ders.: Collected Poems. Hrsg. von Edward Mendelson. New York: Vintage International, 1991. S. 878.

Bargmann, Wolfgang: Das Zwischenhirn-Hypophysensystem. Berlin: Springer, 1954.

Benn, Gottfried: Beitrag zur Geschichte der Psychiatrie [1910]. In: ders.: Sämtliche Werke. Bd. 3. Hrsg. von Gerhard Schuster. Stuttgart: Klett-Cotta, 1987. S. 7-10.

Birtsch, Nicole: Orientierungsversuche im Niemandsland zwischen Medizin und Poetik. Das Verhältnis von Körpergedächtnis und Poesie in den Texten von Durs Grünbein. In: Einschnitte: Identität in der Moderne. Hrsg. von Oliver Kohns und Martin Roussel. Würzburg: Königshausen \& Neumann, 2007. S. 103-115.

Choudhury, Suparna, und Jan Slaby (Hrsg.): Critical Neuroscience. A Handbook of the Social and Cultural Contexts of Neuroscience. Chichester: Blackwell, 2012.

Dürbeck, Gabriele „Wenn es stimmt, dass wir schwierige Tiere sind“. Anatomie und Anthropologie in Durs Grünbeins „Mensch ohne Großhirn“. In: Zeitschrift für Germanistik 19.1 (2009). S. 133-145.

89 Durs Grünbein: Der cartesische Taucher. Drei Meditationen. Frankfurt a. M.: Suhrkamp, 2008. S. 90. Vgl. hierzu auch Laak: Bilder der Wissenschaft, S. 138-142. 
Eibl, Karl, Katja Mellmann und Rüdiger Zymner (Hrsg.): Im Rücken der Kulturen. Paderborn: Mentis, 2007.

Ertel, Anna Alissa: Körper, Gehirne, Gene. Lyrik und Literaturwissenschaft bei Ulrike Draesner und Durs Grünbein. Berlin: De Gruyter, 2010.

Fioretos, Aris: Mein schwarzer Schädel. Berlin: DAAD Berliner Künstlerprogramm, 2003. Wieder abgedruckt in: Aris Fioretos: Das Maß eines Fußes. München: Hanser, 2008. S. 202-249.

Fleck, Ludwig: Entstehung und Entwicklung einer wissenschaftlichen Tatsache. Frankfurt a. M.: Suhrkamp, 1980.

Fromm, Waldemar: Die Literatur und das Gehirn: zur Rezeption der Naturwissenschaften bei Botho Strauß und Durs Grünbein. Ein Vergleich. In: Kulturpoetik 9.2 (2009). S. 233-251.

Fuchs, Thomas: Das Gehirn - ein Beziehungsorgan. Eine phänomenologisch-ökologische Konzeption. 4. akt. und erw. Auflage. Stuttgart: Kohlhammer, 2013.

Geisenhanslüke, Achim: ,Mensch ohne Großhirn‘. Durs Grünbein und das Ende der Utopien? In: Die eigene und die fremde Kultur. Exotismus und Tradition bei Durs Grünbein und Raoul Schrott. Tagungsprotokolle. Hrsg. von Dieter Burdorf. Iserlohn: Institut für Kirche und Gesellschaft, 2004. S. 63-78.

Grage, Joachim: Stockholm gris - Gehirn, Stadt und Text bei Aris Fioretos und Durs Grünbein. In: Skandinavistik 34.2 (2004). S. 133-149.

Grünbein, Durs: Schädelbasislektion. Gedichte. Frankfurt a. M.: Suhrkamp, 1991.

Grünbein, Durs: Katze und Mond. In: ders.: Galilei vermißt Dantes Hölle und bleibt an den Massen hängen. Frankfurt a. M.: Suhrkamp, 1996. S. 55-60.

Grünbein, Durs: Unter uns Hunden. In: Aris Fioretos: Mein schwarzer Schädel. Berlin: DAAD Berliner Künstlerprogramm, 2003 [Spurensicherung, Bd. 14]. S. 57-61.

Grünbein, Durs: Mein babylonisches Gehirn. In: ders.: Gedicht und Geheimnis. Frankfurt a. M.: Suhrkamp, 2007. S. 19-33.

Grünbein, Durs: Der cartesische Taucher. Drei Meditationen. Frankfurt a. M.: Suhrkamp, 2008.

Grünbein, Durs: Limbische Akte. Gedichte. Ausgew. und mit einem Nachwort versehen von Norbert Hummelt. Stuttgart: Reclam, 2011.

Grünbein, Durs, und Michael Hagner: Hirnbilder und Denkschriften. Ein einführender Dialog. In: Hirngespinste. Denkprozesse zwischen Störung, Genialität und Fiktionalität in Künsten und Wissenschaften. Hrsg. von Wladimir Velminski. München: Fink, 2013. S. 21-45.

Hasler, Felix: Neuromythologie. Eine Streitschrift gegen die Deutungsmacht der Hirnforschung. Bielefeld: transcript, 2012.

Henriksen, Gjermund, und Frode Willoch: Imaging of opioid receptors in the central nervous system. In: Brain: Journal of Neurology 131 (2008). S. 1171-1196.

Herrmann, Karin (Hrsg.): Neuroästhetik. Perspektiven auf ein interdisziplinäres Forschungsgebiet. Kassel: University Press, 2011.

Hess, Walter R.: Das Zwischenhirn. Syndrome, Lokalisationen, Funktionen. 2. Auflage. Basel: Schwabe, 1952.

Joney, Terry, und Eugenii Rabiner: The development, past achievements, and future directions of brain PET. In: Journal of Cerebral Blood Flow \& Metabolism 32 (Juli 2012). S. 1426-1454.

Koepsell, Kilian, und Carlos Spoerhase: Neuroscience and the Study of Literature. Some Thoughts on the Possibility of Transferring Knowledge. In: Journal of Literary Theory 2 (2008). S. 363-374.

Laak, Lothar van: Die Bilder der Wissenschaft - die Bilder der Literatur. Das interdisziplinäre Potential der Einbildungskraft im Blick von Eichendorff und Grünbein. In: Literaturwissen- 
schaft interdisziplinär. Hrsg. von Lothar van Laak. Heidelberg: Synchron Wissenschaftsverlag der Autoren, 2010. S. 131-142.

Lauer, Gerhard: Das Spiel der Einbildungskraft. Zur kognitiven Modellierung von Nachahmung, Spiel und Fiktion. In: Literatur als Spiel. Evolutionsbiologische, ästhetische und pädagogische Konzepte. Hrsg. von Thomas Anz und Heinrich Kaulen. Berlin: De Gruyter, 2009. S. 27-37.

Meyer, Anne-Rose: Physiologie und Poesie. Zu Körperdarstellungen in der Lyrik von Ulrike Draesner, Durs Grünbein und Thomas Kling. In: Gegenwartsliteratur. Ein germanistisches Jahrbuch 1 (2002). S. 107-133.

Monyer, Hannah u. a.: Das Manifest. Elf führende Neurowissenschaftler über Gegenwart und Zukunft der Hirnforschung. In: Gehirn \& Geist 6 (2004). S. 30-37.

Müller-Tamm, Jutta: WeltkörperInnenraum. Anmerkungen zur literarischen Anthropologie des Körperinneren. In: IASL 25.1 (2000). S. 95-133.

Nieuwenhuys, Rudolf: Chemoarchitecture of the Brain. Berlin: Springer, 1985.

Porath, Erik: Literarische Kranionautik. Erzählweisen des Gehirns. In: Chiffre 2000 - Neue Paradigmen der Gegenwartsliteratur. Hrsg. von Corina Caduff und Ulrike Vedder. München: Fink, 2005. S. 43-56.

Riedel, Wolfgang: Poetik der Präsenz. Idee der Dichtung bei Durs Grünbein. In: IASL 24.1 (1999). S. 82-105.

Roth, Gerhard: Das Gehirn und seine Wirklichkeit. Kognitive Neurobiologie und ihre philosophischen Konsequenzen. Frankfurt a. M.: Suhrkamp, 1994.

Schrott, Raoul, und Arthur Jacobs: Gehirn und Gedicht. Wie wir unsere Wirklichkeiten konstruieren. München: Hanser, 2011.

Simeons, Albert T. W.: Das tyrannische Gehirn als Ursache seelisch bedingter Leiden. Rüschlikon-Zürich: A. Müller, 1962.

Singer, Wolf: Keiner kann anders, als er ist. http://www.faz.net/aktuell/feuilleton/ hirnforschung-keiner-kann-anders-als-er-ist-1147780.html. Frankfurter Allgemeine Zeitung Online, 8. Januar 2004 (14. April 2015).

Singer, Wolfgang: Selbsterfahrung und neurobiologische Fremdbestimmung. Zwei konfliktträchtige Erkenntnisquellen. In: Deutsche Zeitschrift für Philosophie 52 (2004). S. 235255.

Slaby, Jan: Perspektiven einer kritischen Philosophie der Neurowissenschaften. In: Deutsche Zeitschrift für Philosophie 59 (2011). S. 375-390.

Snyder, Solomon H.: Chemie der Psyche. Drogenwirkungen im Gehirn [Drugs in the Brain, 1986]. Heidelberg: Spektrum der Wissenschaft, 1988.

Stier, Markus: Ethische Probleme in der Neuromedizin. Identität und Autonomie in Forschung und Diagnostik. Frankfurt a. M.: Campus 2006.

Stockhorst, Stefanie: Ästhetisierung der Anatomie. Medizinische und literarische Referenzräume in Durs Grünbeins ,Schädelbasislektionen‘. In: Schreiben am Schnittpunkt. Poesie und Wissen bei Durs Grünbein. Hrsg. von Kai Bremer, Fabian Lampert und Jörg Wesche. Freiburg: Rombach, 2007. S. 191-212.

Weigel, Sigrid: Literatur als Voraussetzung der Kulturgeschichte. Schauplätze von Shakespeare bis Benjamin. München: Fink, 2004.

Welsh, Caroline: Die „Dunkelheit hinter dem Stirnportal“. Begegnungen von Literatur und Hirnforschung. In: Hirngespinste. Denkprozesse zwischen Störung, Genialität und Fiktionalität in Künsten und Wissenschaften. Hrsg. von Wladimir Velminski. München: Fink, 2013. S. 63-80. 